\title{
The justification of interaction of fruits with the rolling-in device of a combine-harvester for harvesting cucurbit crops
}

\author{
Maxim Ulyanov ${ }^{1, *}$, Vitaly Ceplyaev ${ }^{1}$, Michael Shaprov ${ }^{1}$, and Nikolay Ayugin ${ }^{2}$. \\ ${ }^{1}$ Volgograd State Agrarian University, 26, Universitetskiy Avenue, Volgograd, 400002, Russia \\ ${ }^{2}$ Ulyanovsk State Agrarian University named after P.A. Stolypin, 432017 Ulyanovsk, Russia
}

\begin{abstract}
The role of cucurbit crops in the life of man has been studied and presented. The process of interaction between the rolling-in device of a combine-harvester and a fruit, as well as the process of hitting water-melons into different surfaces, have been considered. The diagram of the distribution of velocities when the rolling-in device interacts with a fruit, as well as the area of expansion of plastic deformation as a result of the impact of a fruit, have been presented. The equation of work, which arises when a fruit hits into the rolling-in device, has been considered, the work being decomposed into plastic work of deformation and elastic work of deformation. The work of plastic deformation, which arises when fruits come into collision with a surface, is considered in more detail, the work being decomposed into plastic work of deformation in the volume, which is limited by a spherical segment and a cone. The absolute and allowable velocities after the impact have been theoretically determined and substantiated. The parameters of allowable velocities for the varieties "Crimson sweet" and "Kholodok" have been theoretically found and the confirmation of the theoretical analysis was carried out in experimental research, which was conducted on a laboratory installation for determining the critical speed of impact on fruits of cucurbit crops. The comparison of the theoretical and experimental values of the terminal velocities confirms the credibility of the research.
\end{abstract}

\section{Introduction}

Water-melons are considered to be the largest berries in the vegetable world. As any of the agricultural materials, a water-melon has a complex texture, which is represented, from a physical standpoint, by three main components: solid, liquid, and gaseous. The liquid is the base of its mechanically weak pulp, which is covered with a smooth elastic membrane. The form of a melon which occurs most frequently is spherical; from a mathematical standpoint - close to spherical. Such a form is characterized by essential values of surface stresses. Practically, this statement is confirmed by the phenomena of a rind break by the forces of surface tension, which are of explosive nature even when slight mechanical impacts are applied to a water-melon. A break is accompanied with deep fractures, sometimes down to the fruit center; the fracture lines are of stochastic nature in the length and directions. If we refer to the fruit build, one should note that the liquid therein, which is in the form of a water solution with other elements, sucrose, fructose, etc., constitutes up to $90 \%$ of the whole weight. Thereby, the liquid is in a free state. It appears from this that the liquid in a water-melon, when an external mechanical impact is applied, can be a source of internal breaking forces of shock or wave types. The hydraulic effect is taken as a basis of formation of these forces. In their value, these forces considerably exceed the forces of external impact on a fruit $[1,2]$.

When a visual observation is carried out, with the practical conclusions being also taken into account, one should note that the majority of the fruits damaged occur due to impact loads. Hence, it appears that the basic constituent of the dynamic loads on water-melon fruits is a shock. The theoretical study of this phenomenon enables to determine the basic cinematic and design parameters for water-melon harvesters [3].

However, to obtain more consistent results, it is necessary to study the physical and mechanical properties. The mathematical models to describe the shock process will be more consistent if the theory of study of various media is used for the induction thereof [4].

\section{Results}

Thus, the material deformation process, when dynamic loading is applied, should be considered by assimilating it to a certain mechanical model of a fruit.

Thereby, the liquid is encased in the cell membranes, but they are unable to resist even minor mechanical impacts and virtually break even after a low effort. Seeds, however, form part of a water-melon pulp, and they are covered with film membranes with air inside. The total volume of such inclusions can reach up to $5 \%$ of a fruit weight, consequently, up to $7-8 \%$ in a single

* Corresponding author: ulyanovmv@mail.ru 
pulp. I. S. Yegorov determined that the relaxation time after a shock impact on a water-melon fruit reaches $0.01 \ldots 0.035 \mathrm{~s}$. The relaxation time is a period during which a wave passes from the striker to the rind and comes back [5].

The subsequent experiments have established that the assumption of a shock impact on a fruit is confirmed by the conclusions about the relaxation.

The question of interaction between the working tool and a fruit has been considered by many researchers, and the process of hitting water-melons into different surfaces, which takes place in operation of some collecting units, has been also studied in close detail. We have proposed the study of this phenomenon in respect to the collector.

A shock impact takes place in the contact point between the working tool and a motionless water-melon fruit. In mechanics, this phenomenon is described with the equation:

$$
m u-m v=\int_{0}^{\tau} F d t=S,
$$

where: $v$ is the speed of the material point before the shock impact and $u$ is its speed after the shock impact.

In our case, a water-melon which is influenced by the working tool with certain speed $v$ is considered to be the material point.

If the surface of the working tool, which is a plank installed under a certain angle, did not rotate, the impact speed in the contact would equal its motion speed. And, since the working tool rotates with a certain frequency in the collector structure and alongside it, it moves translationally, there arise relative and transportation velocities, that is, the cumulative value of the impact speed is equal to the vector sum of the speeds:

$$
\bar{v}_{a}=\bar{v}_{e}+\bar{v}_{r},
$$

where: $\bar{v}_{a}$ is the vector of the absolute motion speed of the working tool, $\bar{v}_{q}$ is the vector of the transportation velocity, $\bar{v}_{w}$ is the vector of the relative velocity.

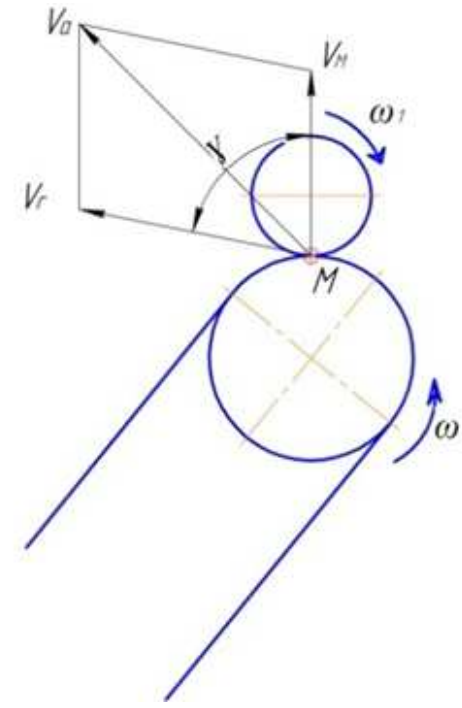

Fig. 1. The diagram of the speed distribution when the working tool interacts with a fruit

Transportation velocity $v_{e}$ equals collector motion speed $v_{e}=v_{\mathrm{M}}$. The relative velocity is the peripheral speed of the working tool point, which is simultaneously the point of contact with the fruit.

Let us denote the correlation between the transportation and relative velocities through coefficient $\lambda, \lambda=\frac{v_{g}}{v_{r}}$. To determine the absolute increment speed after the shock impact, let us consider the diagram in fig. 1.

According to mechanics [6]:

when a shock is direct

$$
v_{a}=\left[v_{o}^{2}+v_{e}^{2}\right]^{1 / 2}
$$

when a shock is oblique

$$
v_{a}=\left(v_{e}^{2}+v_{r}^{2}+2 v_{e} v_{r} \cos \gamma\right)^{1 / 2} \text {. }
$$

Let us replace the transportation velocity with its value:

$$
\begin{aligned}
& v_{a}=\left(\lambda^{2} v_{r}^{2}+v_{r}^{2}+2 v_{r} v_{r} \lambda \cos \gamma\right)^{1 / 2}=v_{r}\left(\lambda^{2}+\right. \\
& 2 \lambda \cos \gamma+1)
\end{aligned}
$$

Let us denote $\left(\lambda^{2}+2 \lambda \cos \gamma+1\right)^{1 / 2}=\Lambda$, then $v_{a}=\Lambda v_{\text {w. }}$. We consider that a fruit rolls over without gliding under the influence of the working tool.

Shock phenomena arise in the contact point. V.P. Goryachkin noted that any shock phenomena are inextricably linked with the vibrations of elastic bodies [1]. These vibrations are decaying and, in some cases (a minor shock pulse or a high density of the material), even the first shock wave does not come back.

Many researchers characterize the internal mechanics of a shock with two stages. The first stage is the expansion of the compression wave and this stage finishes when the speeds of any internal particles become zero. The second stage is the expansion of the tension wave, which finishes when the speeds of any particles are equal to $v$ (an absolutely elastic shock) [7].

Thus, a body bounces from the striker with speed $v$, which is equal to the striker motion speed at the end of the second stage where an absolutely elastic shock takes place. The whole energy obtained during the shock is consumed for the recovery of its shape and the value for the resilience is numerically equal to $W=\frac{m v^{2}}{2}$, where $m$ is the body weight, $v$ is the striker motion speed. The phenomenon of an incompletely elastic shock is characteristic of the most agricultural processes, with part of energy being lost for the following motion and, according to the expression by V.P. Goryachkin, composing "the dead part of the shock" [1]. The impact energy is thereby consumed for the plastic deformation and resilience. Identical phenomena take place in cucurbit fruits under a mechanical impact [1]. In conformity with the tests, the plastic deformation involves some deformable volume (fig. 2). 


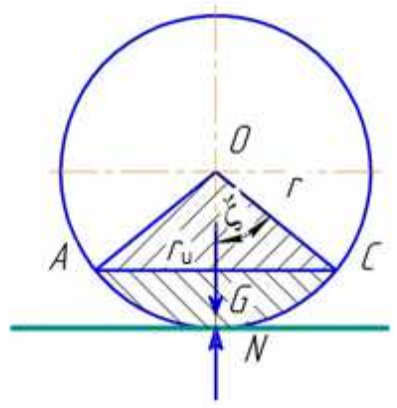

Fig. 2. The area of the expansion of plastic deformation when a fruit is hit

To solve the problem of impacting a water-melon with an absolutely rigid body, it is necessary to proceed to discrete models [8]. The discrete model for a viscoelastic medium (Kelvin-Feucht medium) represents a successive combination of elastic and plastic elements. The rheological equation is written in the form of:

$$
\sigma=E \varepsilon+\mu \frac{d s}{d t}
$$

where: $E$ is the elastic modulus, $\boldsymbol{\varepsilon}$ is the linear strain of the body, $\boldsymbol{\mu}$ is the viscosity coefficient.

The first summand of the equation describes the elastic properties of the body; the second summand describes the plastic ones. In a manner similar to this equation, one can write down the energy equation during a shock impact:

$$
A=W_{1}+W_{2}
$$

where: $W_{1}$ is the elastic work of deformation, $W_{2}$ is the plastic work of deformation.

The value of work in general, which is obtained during a shock impact, can be written with the equation:

$$
A=\int_{0}^{t} F v \cos \varphi d t
$$

where: $\mathrm{F}$ is the sum of the forces, which impact the system, $v$ is the system joint speed, $\varphi$ is the angle between the sum vector of the forces and speeds according to the diagram (fig. 1 ) $\varphi=\gamma=0,73 \mathrm{rad}$.

The value of the elastic work of deformation of a fruit rind is expressed as:

$$
\frac{m\left(v_{\mathrm{a}}+\Delta v\right)^{2}}{2}-\frac{m v^{2}}{2}=m v \Delta v+\frac{m(\Delta v)^{2}}{2}
$$

where: $v_{\mathrm{a}}$ is the absolute speed of point " $\mathrm{M}$ " of the working tool, which is the point of contact with a fruit; $\Delta v$ is the increment velocity due to the resilience.

By substituting expressions (5) and (6) into equation (4) and taking into account that $v_{a}=\wedge v_{r}$, we will obtain the following expression:

$$
A \int_{0}^{t} F v_{r} d t=m k v_{r} v_{r}+0,5 m k^{2}\left(v_{r}\right)^{2}+W_{2}
$$

For an absolutely elastic shock, we have the expression:

$$
\begin{aligned}
& \mathrm{A} \int_{0}^{t} m a v_{r} d t=m \int_{0}^{v_{r}} v_{r} d v_{r}+ \\
& 0,5 m \int_{0}^{v_{r}} d\left(v_{r}\right)^{2}
\end{aligned}
$$

$$
\wedge \int_{0}^{t} m a v_{r} d t=m k v_{r}^{2}+0,5 m k^{2} v_{r}^{2}+W_{2} \text { (9) }
$$

where $k$ is the numerical coefficient, which is called a coefficient of a body restitution [9]. It is determined experimentally and is numerically equal to the ratio between a body speed after the impact and the speed of the striker. The coefficient of a body restitution for water-melons equals $\mathrm{k}=0.38$. Let us determine the part of energy consumed for plastic deformation during a shock impact from the equation:

$$
W_{2}=\int_{0}^{2} p \mathrm{fdl}
$$

where: $p$ is the specific deformation force in a shock destruction, $\mathrm{N} / \mathrm{mm}^{2}, \quad$ is section area, $\mathrm{mm}^{2}, l$ is the expansion depth of the deformation, $\mathrm{mm}$.

According to the theory, plastic expression (10) represents the most general expression of work of deformation [1]. For an accurate mathematical description of body impact processes, the equilibrium equation of an infinitely small medium volume is applied, the solution of which is difficult in most cases. Let us introduce some assumptions to simplify the mathematical expression:

1. Any sections of the body, which were formed before a shock impact, hold their shape during the shock impact as well;

2. The normal stresses are equal between each other in any cross section points;

3 . Due to a negligible friction coefficient on a watermelon rind $\mathrm{f}=0.2$, we do not take into account the shear stress on the fruit surface.

On the basis of the law of least resistance, the movement of body points during a shock impact takes place towards the least resistance and in the direction of the shock impact. Hence, we conclude that the plastic deformation develops when a fruit is hit in the volume, which is limited by a spherical segment and a cone (fig. 2) with the base in the form of a circle with radius $r$.

Considering that the body volume before and after the shock impact does not change, we will express the square of the base through the volume: $F=\frac{V}{l}$, where $\mathrm{V}$ is the constant volume of the body part, which is subject to a shock pulse $\mathrm{m}^{3} ; l$ is the deformation depth, $\mathrm{m}$. Then the complete plastic work of deformation is:

$$
W_{2}=W_{2}^{\prime}+W_{2}^{\prime \prime}
$$

where: $W_{2}^{\prime}$ is the plastic work of deformation in the volume limited by the spherical segment; $W_{2}^{\prime \prime}$ is the plastic work of deformation in the volume limited by the cone.

$$
\begin{aligned}
& W_{2}^{\prime}=V_{u} \int p \frac{d l}{l}=\left(3 r_{\sigma}-l\right) \frac{\pi l^{2}}{3} \int \frac{d l}{l} ; \\
& l_{u}=r-r \cos \xi ; d l_{u}=r_{u} \sin \xi d \xi ;
\end{aligned}
$$

where: $\xi$ is the half of the angle between the deformation areas.

Let us substitute expressions $l_{\mathrm{u}}$ and $\mathrm{dl}_{\mathrm{u}}$ into formula (14) and, by replacing the limits, we will come to the following expression:

For an elastoplastic (incompletely elastic) shock: 


$$
\begin{aligned}
& W_{2}^{\prime}=\pi r_{u}^{3} \frac{\cos ^{3} \xi-3 \cos \xi+2}{3} \int_{0}^{\xi} \frac{\sin \xi}{1-\cos \xi} p d \xi= \\
& \left.\pi r_{u}^{3} \frac{\cos ^{3} \xi-3 \cos \xi+2}{3} \ln |1-\cos \xi|\right|^{+\xi} x p
\end{aligned}
$$

Here $p$ is a variable and it depends on the depth, but minor fluctuations from the average value do not influence the final result. Therefore, we factor $p_{s r}$ outside the integral sign in the equation.

$$
W_{2}^{\prime}=\left.p_{s r}^{\prime} \pi r_{u}^{3} \frac{\cos ^{2} \xi-3 \cos k^{2}+2}{3} \ln |1-\cos \xi|\right|_{0} ^{+\xi}
$$

We will express the plastic work of deformation in the cone volume respectively:

$$
\begin{aligned}
W_{2}^{\prime \prime} & =V_{k} \int p \frac{d l}{l}=\frac{1}{3} \pi r_{k}^{2} l \int \frac{d l}{l} p, \\
l_{k} & =r_{k} \cos \xi, d l_{k}=-r_{k} \sin \xi d \xi .
\end{aligned}
$$

where $V_{k}$ is the rate of development of the plastic deformation in the cone volume $\mathrm{m} / \mathrm{s} ; r_{k}$ is the cone radius, $\mathrm{m} ; l_{k}$ is the deformation depth in the cone volume, $\mathrm{m}$.

By substituting values $l_{k}$ and $d l_{k}$ obtained into expression (15), we have:

$$
\begin{aligned}
& W_{2}^{\prime \prime}=-p_{s r}^{\prime \prime} \frac{1}{3} \pi r_{k}^{3} \cos \xi \int_{0}^{+\xi} \frac{\sin \xi d \xi}{\cos \xi}= \\
& \left.\frac{1}{3} p_{s r}^{\prime \prime} \pi r_{k}^{3} \cos \xi \ln |\cos \xi|\right|_{0} ^{+\xi}= \\
& \left|p_{s r}^{\prime \prime} \frac{1}{3} \pi r_{k}^{3} \cos \xi \ln \right| \sec \xi||_{0}^{+\xi} \mid
\end{aligned}
$$

By substituting values $W_{2}^{\prime}$ and $W_{2}^{n}$ in formula (5), we will obtain:

$$
\begin{aligned}
& W_{2}=p_{s r}^{r} \pi r_{u}^{3} \frac{\cos ^{2} \xi-3 \cos \xi+2}{3} \ln |\mathbf{1}-\cos \xi|+ \\
& p_{s r}^{\prime \prime} \frac{1}{3} \pi r_{k}^{3} \cos \xi \ln |\sec \xi|
\end{aligned}
$$

The first summand of the expression obtained is nothing else than the plastic work of deformation consumed for the destruction of a fruit rind; the second one is the energy consumed for the destruction of the pulp. Value $\mathbf{p}_{s r}$ for the rind will be considerably greater than that for the pulp. As we have arranged that the friction on the fruit surface is very low, specific pressure $\mathrm{p}_{\mathrm{cp}}$ will equal to the yield stress, i.e. $\mathrm{p}_{s r}=\sigma_{z}$, where $\sigma_{s}$ is the yield stress [11, 12]. This value can be determined on the basis of the equation of the distribution of normal stress on the contact surface in the absence of friction, which was proposed by Tomlenov A.D.

$$
\sigma_{N}=\sigma_{s}\left(1+\frac{\pi}{2}-\gamma\right),
$$

where: $\sigma_{N}$ is the normal stress which arises when a material is plastic, $\mathrm{N} / \mathrm{m}^{2} ; \gamma$ is the angle between the axis of the sphere being impressed in and the line from the center to the contact point between the sphere and the material surface; $\sigma_{s}$ is the shearing yield stress of the material, $\mathrm{N} / \mathrm{m}^{2}$.

When a sphere with certain diameter $d_{s}$ is impressed in for the extreme event - when the sphere is impressed for the maximum diameter, we have:

$$
P=1.8 \pi r_{s}^{2} \sigma_{s}
$$

where: $r_{s}$ is the sphere's radius; $\mathrm{P}$ is the pressure force.

$$
\sigma_{s}=\frac{P}{1,8 \pi r_{z}^{2}}
$$

Let us substitute value $\sigma_{s}$ into formula (17):

$$
\begin{aligned}
& W_{2}=\frac{\cos ^{3} \xi-3 \cos \xi+2}{3} \sigma_{s}^{3} \pi r_{s}^{3} \ln |1-\cos \xi|+ \\
& \frac{\cos \xi}{3} \sigma_{s}^{\prime \prime} \pi r_{k}^{3} \ln |\sec \xi| \\
& W_{2}=\frac{\cos ^{2} \xi-3 \cos \xi+2}{3} \frac{F_{l}}{1,8 \pi r_{k}^{2}} \pi r_{z}^{3} \ln |1-\cos |+ \\
& \frac{\cos \xi P_{k}}{3} \pi r_{g}^{3} \pi r_{k}^{3} \ln |\sec \xi|
\end{aligned}
$$

When substituting the respective numerical values into expression obtained (19), we come to the conclusion that the multiplier of the first fraction $\frac{\cos ^{3} \xi-3 \cos \xi+2}{3} \rightarrow 0$, and therefore, the whole first summand will tend to zero. Then the plastic work of deformation is determined by the formula:

$$
W_{2}=\frac{\cos z}{3} \frac{F_{h t}}{1,8 r_{g}^{2}} r_{k}^{3} \ln |\sec \xi| \text {. }
$$

The complete impact energy:

$$
\begin{aligned}
& A=\wedge m v_{r}^{2}=m k v_{r}^{2}+0,5 m k^{2} v_{r}^{2}+ \\
& \frac{\cos k}{3} \frac{P_{k}}{1,8 r_{g}^{2}} \gamma_{k}^{3} \ln |\sec \xi|
\end{aligned}
$$

Let us determine the allowable impact speed of the working tool on a water-melon fruit from the expression obtained.

$$
v_{d} \leq\left[\frac{P_{k} r_{h}^{2} \cos \xi_{d} \ln \left|\sec F_{d}\right|}{5,4 r_{3}^{2}\left(\sin -k m-0,5 m k^{2}\right)}\right]^{\frac{1}{2}}
$$

Numerical methods have been used to solve the expression obtained. To that end, a program was made in Mathcad system and, by substituting unknown values $(\mathrm{m}, \xi)$, the allowable speed forces have been determined for water-melon fruits of varieties: "Crimson sweet" and "Kholodok". The parameters of the allowable speeds theoretically found for variety "Crimson sweet" are at most $1.2 \mathrm{~m} / \mathrm{s}$, and those for variety "Kholodok" are 1.4 $\mathrm{m} / \mathrm{s}$. Such a difference is explained by the strength of the armor, which is of great importance for "Kholodok" water-melons.

The experimenal research to determine and verify the theoretical analysis, which had been carried out earlier, was performed on special equipment set out in fig. 3 .

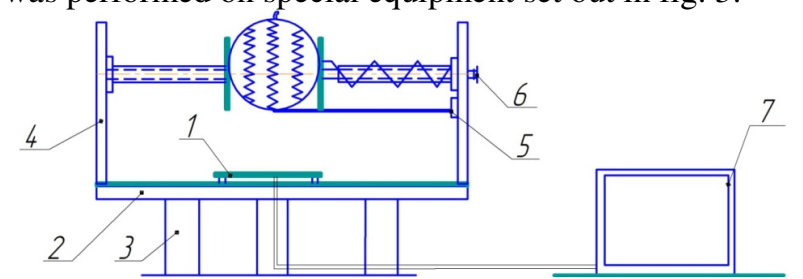

Fig. 3. The installation to determine the critical speed of impact on a fruit: 1 - object table; 2 - base; 3 - supports; 4 - stands; 5 fixing arrow; 6 - spring arm-fixture; 7 - computer.

Water-melon fruits were dropped on a special table in the form of a hard metal plate equipped with a sensor, which was connected to a computer. When a fruit fell on the table from a certain fixed height, the sensor transmitted an impulse to the computer. A diagram of an 
impact pulse, which referred to the fruit fall time, was built according to the values received.

However, the most justified test results from the practical standpoint have been obtained concerning the storage period of water-melon fruits, which were subject to a shock impact. The diagram of the change in the fruit storage period after a shock impact is shown in fig. 4 .

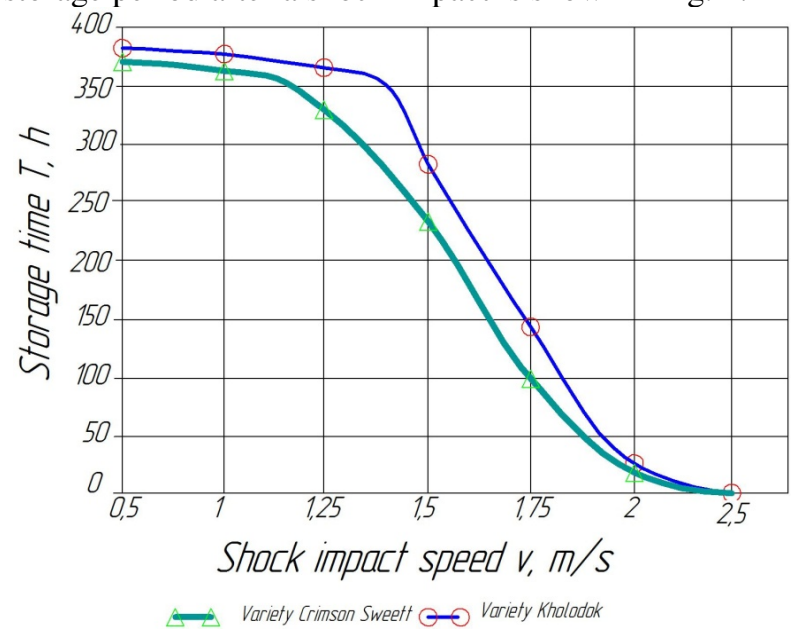

Fig. 4. The diagram of a change in a fruit storage period depending on the impact speed

As reflected by a characteristic change in the curves set out in the diagram, they represent a parabolic dependence. It should be noted that the parameters of the allowable speed are $1.0 \mathrm{~m} / \mathrm{s}$ for variety "Crimson sweet" and $1.3 \mathrm{~m} / \mathrm{s}$ for variety "Kholodok".

\section{Conclusion}

On the basis of the theoretical analysis of the interaction between the working tool and a water-melon fruit, it has been established that an elastoplastic deformation develops in a water-melon fruit in the contact point after an impact of the working tool. On the basis thereof, the dependences of the expansion of the elastic and plastic deformations have been established. The equations obtained analytically enable to determine the allowable impact speed of the working tool on a fruit, which equals to $1.2 \mathrm{~m} / \mathrm{s}$ for "Crimson sweet" fruits and $1.4 \mathrm{~m} / \mathrm{s}$ for "Kholodok" fruits.

The subsequent experimental research in regard to the fruit storage periods has confirmed the theoretical parameters obtained and, as reflected by the curves, the speed limits for both varieties set out in diagram (fig.4) correlate as follows: "Crimson sweet" $-1.0 \mathrm{~m} / \mathrm{s}$, "Kholodok" $-1.3 \mathrm{~m} / \mathrm{s}$. The comparison of the theoretical and experimental speed limit values confirms the accuracy of the research.

\section{References}

1. A. Ovchinnikov, A. Tseplyaev, M. Shaprov, M. Ul'yanov, S. Poltorynkin, A. Sedov, V. Tseplyaev, V. Slavutsky, V. Berdyshev, V. Bocharnikov, I. Borisenko, S. Grigorov, S. Fomin, V. Ol'garenko, The choice of technology of agricultural crops cultivation on the basis of the manual labor costs indicator optimization (for the example of vegetable and melon crops cultivation), ARPN Journal of Engineering and Applied Sciences, 14(22), 33897-3905 (2019)

2. M. Ulyanov, A. Ulyanov, The results of laboratory and factory tests of an active type swath maker for cucurbit crops, Bulletin of Orenburg State Agrarian University, 3(31), 98-100 (2011)

3. A. Roshanianfard, N. Noguchi, T. Kamata, Design and performance of a robotic arm for farm use, International Journal of Agricultural and Biological Engineering, 12(1), 146-158 (2019)

4. X. Xing, F. Liu, J. Gao, Design and implementation of watermelon traceable identification algorithm based on biometric texture information, Nongye Gongcheng Xuebao Transactions of the Chinese Society of Agricultural Engineering, 33, 298-305 (2017)

5. S. Sakai, K. Osuka, M. Umeda, Use of a heavy material handling agricultural robot for harvesting watermelons, Proceedings of the International Conference on Automation Technology for Off-road Equipment, ATOE (2004)

6. S. Giwa, T. Akanbi, Mechanization of melon processing and novel extraction technologies: A short review, Scientific African, 9, e00478 (2020)

7. H. Guo, M. Wang, Y. Dong, Z. Xie, Design and experiment for the header of seed melon combine harvester, International Agricultural Engineering Journal, 29(2), 78-85 (2020)

8. Abidzar H. Tawakal, A. Prayoga, The Development of Methods for Detecting Melon Maturity Level Based on Fruit Skin Texture Using the Histogram of Oriented Gradients and the Support Vector Machine, Proceedings of 2019 4th International Conference on Informatics and Computing, ICIC, 8985953 (2019)

9. U. Ahmad, D. Bermani, P. Mardison, Color distribution analysis for ripeness prediction of Golden Apollo Melon, Telecommunication Computing Electronics and Control, 16(4), 16591666 (2018)

10. H. Paris, Y. Tadmor, A. Schaffer, Cucurbitaceae Melons, Squash, Cucumber, Encyclopedia of Applied Plant Sciences, 3, 209-217 (2016)

11. M. Mann, B. Zion, I. Shmulevich, D. Rubinstein, Determination of robotic melon harvesting efficiency: A probabilistic approach, International Journal of Production Research, 54(11), 3216-3228 (2016)

12. M. Mann, B. Zion, I. Shmulevich, D. Rubinstein, R. Linker, Combinatorial Optimization and Performance Analysis of a Multiarm Cartesian Robotic Fruit Harvester-Extensions of Graph Coloring, Journal of Intelligent and Robotic Systems: Theory and Applications, 82(3-4), 399-411 (2016) 